\title{
Reversible cerebral vasoconstriction syndrome associated with autonomic dysreflexia
}

\author{
Bengt Edvardsson - Staffan Persson
}

Received: 6 December 2009/ Accepted: 1 February 2010/Published online: 26 February 2010

(c) Springer-Verlag 2010

\begin{abstract}
A 32-year-old man with a residual spastic quadriparesis from a traumatic C5-C6 fracture experienced a severe thunderclap headache. The medical history revealed an episode of autonomic dysreflexia (AD) due to neurogenic bladder/urinary tract infection (UTI). Blood pressure monitoring at admission revealed hypertension; blood pressure reaching 160/100 $\mathrm{mmHg}$ (average blood pressure in these patients and also in this patient being 90/ $60 \mathrm{mmHg}$ ). CT scan of the head, cerebrospinal fluid examination, CT angiography and MR angiography of the brain vessels were normal. Another UTI and a subsequent spell of $\mathrm{AD}$ were diagnosed. The patient continued to experience recurrent thunderclap headaches. Selective catheter cerebral angiography revealed multiple calibre changes in the intracranial blood vessels. A diagnosis of reversible cerebral vasoconstriction syndrome (RCVS) due to $\mathrm{AD}$ was considered. A magnetic resonance imaging (MRI) of the brain after 2 weeks revealed ischaemic changes in the left hemisphere. Follow-up brain MRI after 3 weeks showed reduction in size of the ischaemic changes, and catheter angiography after 6 weeks demonstrated improvement/normalization. A diagnosis of RCVS could be established. Repeated MRI/CT of the brain after 6 months demonstrated a large infarction in the left hemisphere. RCVS has been reported to occur in various clinical settings. It can occur in the setting of $\mathrm{AD}$ in patients with traumatic cervical cord injury. Prompt recognition of RCVS may be of vital importance to avoid further morbidity in patients with spinal cord injury.
\end{abstract}

B. Edvardsson $(\varangle) \cdot$ S. Persson

Department of Neurology, Faculty of Neurology, Lund University Hospital, 22185 Lund, Sweden

e-mail: Bengt.Edvardsson@med.lu.se
Keywords Reversible cerebral vasoconstriction syndrome - Autonomic dysreflexia - Cerebral infarct . Thunderclap headache · Hypertension .

Reversible posterior leukoencephalopathy syndrome

\section{Introduction}

Reversible cerebral vasoconstriction syndrome (RCVS) has been reported to occur in various clinical settings. Although it may occur spontaneously, various associated conditions have been described, e.g. uncontrolled hypertension, pheochromocytoma, pregnancy or puerperium (postpartum angiopathy), neurosurgical procedures, head trauma, vasoactive substances and recreational drugs. RCVS is a syndrome characterized by a sudden thunderclap-like headache, with or without focal neurological deficits and reversible vascular narrowing of the large and medium-sized cerebral arteries of the circle of Willis and its branches. It occurs most commonly in women aged 20-50 years. Improvement in vasospasm is expected with or without treatment within days to weeks of the onset of symptoms [1-5]. RCVS associated with autonomic dysreflexia $(A D)$ is not hitherto described. We report a patient with spastic residual quadriparesis who developed RCVS with subsequent cerebral infarction in the setting of AD.

\section{Case report}

The patient is a 32-year-old man with a residual spastic quadriparesis from a traumatic C5-C6 fracture ASIA impairment scale (AIS)-B that occurred 12 months prior to hospitalization. After the spinal cord injury (SCI) he 
developed $\mathrm{AD}$, which was noticed during the first hospitalization. The AD episodes included headache, flushing, anxiety and raised blood pressure and were mostly related to the emptying of his neurogenic bladder, coughing and urinary tract infection (UTI). He managed his bladder with self-catheterization. On the day of his second admission, he experienced a sudden severe headache. The pain was located in the left parietal/occipital region and reached a maximum intensity in $<1 \mathrm{~min}$. A thunderclap headache was diagnosed. He also noticed difficulties in finding words. He had a history of headache, nausea and vomiting for the past $24 \mathrm{~h}$. Prior to this event, there was no history of headache. He did not smoke, and he was not using any medication, alcohol or drugs. Physical examination was normal. Vital signs were as follows: blood pressure, $160 / 100 \mathrm{mmHg}$ (average blood pressure in these patients and in this patient being $90 / 60 \mathrm{mmHg}$ ); pulse, 46 beats/ min; respiratory rate, 17 breaths/min; and temperature, $37.1^{\circ} \mathrm{C}$. Sclerae were injected. Fundi were normal. Flushing, anxiety and sweating in the face were noticed. Neurological examination revealed quadriparesis and dysphasia. Laboratory testing revealed a slightly elevated CRP of $35 \mathrm{mg} / \mathrm{l}$, but was otherwise normal (ESR of 11, antineutrophil cytoplasmic antibody, complement levels, serum protein electrophoresis, lupus anticoagulant, syphilis, antinuclear antibody and rheumatoid factor). A CT scan of the head was normal and cerebrospinal fluid (CSF) examination revealed normal opening pressure $\left(170 \mathrm{mmH}_{2} \mathrm{O}\right)$, normal cell count and protein. CT angiography and MR angiography of the brain vessels 2 days after admission showed normal conditions and ruled out intracranial aneurysms, cerebral venous thrombosis and cervical artery dissection. No vasospasm was detected in the large arteries. A UTI and a subsequent spell of AD were diagnosed. The patient received intravenous labetalol $200 \mathrm{mg}$ daily, oral ramipril $5 \mathrm{mg}$ and the antibiotic ciprofloxacin $250 \mathrm{mg}$ two times daily. He continued to experience recurrent thunderclap headaches. Selective catheter cerebral angiography on the ninth day displayed multiple calibre changes in the intracranial blood vessels (Fig. 1). No clinical or laboratory evidence of an underlying vasculitic process could be produced. A diagnosis of RCVS due to $\mathrm{AD}$ was considered. The calcium-channel antagonist nimodipine, $60 \mathrm{mg}$ six times daily, was added on day 9 . The patient's blood pressure was normalized 2 days after the start of nimodipine treatment and the UTI was cured. His thunderclap headache and dysphasia resolved on day 12. MRI of the brain 2 weeks after admission revealed acute ischaemic changes in the left hemisphere (Fig. 1). A follow-up MRI 3 weeks after admission showed reduction in size of the ischaemic changes (Fig. 1). Selective catheter cerebral angiography after 6 weeks displayed regression/ normalization of the multiple calibre changes. The patient was transferred to a rehabilitation centre. Repeated MRI/ CT of the brain after 6 months demonstrated a large infarction in the left hemisphere (Fig. 2). He had no recurrence of thunderclap headache after the first event.

\section{Discussion}

Our case study highlights a patient with AD. Evaluations revealed reversible segmental vasoconstriction of the intracranial blood vessels. RCVS was diagnosed. The patient responded to therapy, but developed a large cerebral infarction.

Patients with RCVS can be misdiagnosed as having primary cerebral vasculitis. However, our patient displayed no signs of vasculitis, e.g. abnormal CSF, abnormal laboratory tests, subacute/chronic onset of headache and insidious/progressive dull aching headache [3].

The only considerable aetiology for RCVS in the patient was AD. Our patient does fulfil the criteria for AD, which is a potentially dangerous complication of subacute and chronic SCI usually occurring with lesions above the splanchnic sympathetic outflow (T6). The most dramatic clinical feature of this syndrome is the sudden significant elevation of blood pressure resulting in a hypertensive emergency. Elevation of blood pressure is perceived by baroreceptors in the aorta, causing increased vagal stimulation by the brain and subsequent distending of rostral vascular beds. The cerebral beds dilate, causing a migrainetype headache. Objectively, any increase in systolic blood pressure greater than $40 \mathrm{mmHg}(130 \mathrm{mmHg})$ should be considered as a possible dysreflexic episode [6].

$\mathrm{AD}$ has been associated with reversible posterior leukoencephalopathy syndrome (RPLS) [7]. In this case report, the authors suggested that RPLS was associated with a sudden elevation of blood pressure due to $\mathrm{AD}$. It has also been associated with hypertensive encephalopathy (HE). Yarkony et al. described three cases of seizures secondary to AD that are compatible with HE [8, 9]. RPLS and $\mathrm{HE}$ are probably the same syndrome, but with different causes [10].

RCVS demonstrates high comorbidity with $\mathrm{HE}$ and RPLS [2, 4]. Vasoconstriction resulting in posterior ischaemic changes is one postulated connection between RCVS and RPLS [2, 11, 12]. The first description of RCVS is in line with the present-day criteria for RPLS [2, 13]. RCVS may possibly occur as a cause of RPLS, due to ischaemia or cerebral oedema and as a consequence of hypertension in RPLS when cerebral autoregulation is overwhelmed by massive increases in arterial and cerebral perfusion pressure [3]. The coexistence of HE/RPLS and RCVS suggests that a disturbance in cerebral arterial tone is the pathophysiologic basis of both syndromes [2, 3, 14]. 
Fig. 1 Selective catheter cerebral angiography (a) 9 days after admission, demonstrating intracranial blood vessels (left hemisphere). MRI, diffusionweighted imaging (DWI) (b) 2 weeks after admission demonstrating acute ischaemic changes in the left hemisphere. Follow-up MRI, DWI (c) and T2-weighted image (d) 3 weeks after admission demonstrating reduction of ischaemic changes multiple calibre changes in the
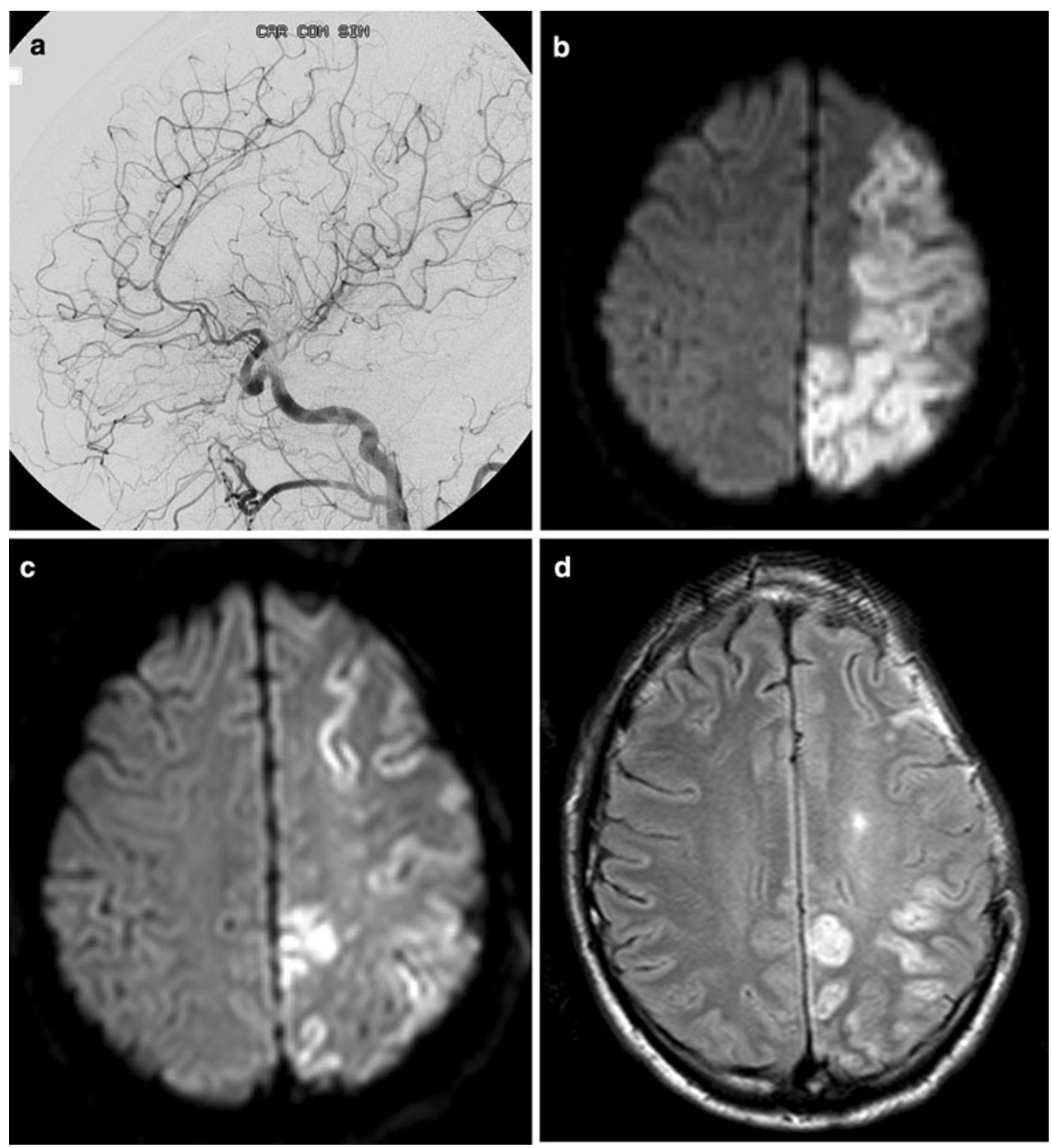

Fig. 2 MRI, T2-weighted image (a) and CT (b) of the brain after 6 months demonstrating a large infarction in the left hemisphere
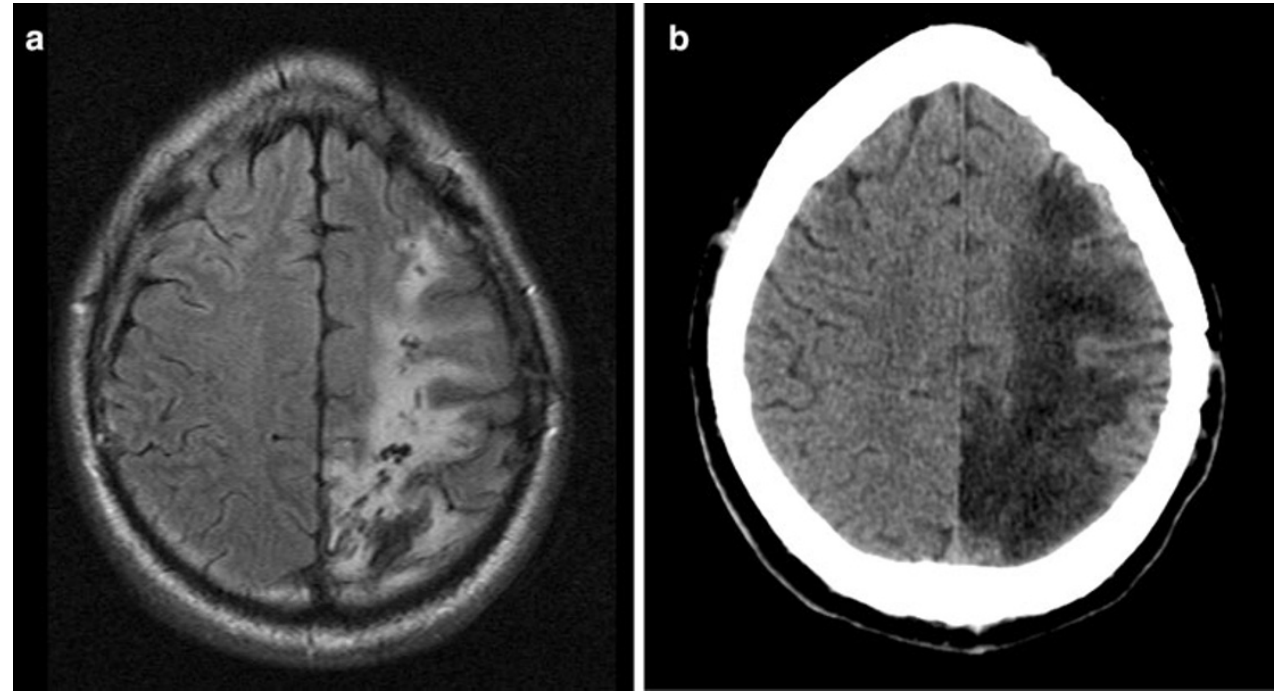

Although the pathophysiology of RCVS remains unknown, the current theory is of a transient disturbance in the control of cerebral vascular tone. Aberrant central regulation of a spontaneous or evoked sympathetic surge may underlie the reversible vascular alterations of RCVS. The theory is derived from the association of RCVS with 
exertion, sympathomimetic agents, pheochromocytoma and hypertensive crises $[2,3,14]$. The temporal pattern of RCVS suggests that the underlying disturbance in the control of cerebral arterial tone first involves small distal arteries responsible for thunderclap headaches, haemorrhages and RPLS and then progresses towards large-sized arteries responsible for ischaemic events as cerebral infarction [1].

In our case, the patient suffered a large stroke. Stroke can be a serious consequence of RCVS. Ducros et al. [1] found that stroke occurred in $9 \%$ of their patients, consistent with the $7 \%$ found in previously published prospective series [15].

There is little evidence of any effective treatment for RCVS [2]. Calcium-channel blockers such as nimodipine are empirically used in the treatment of RCVS. Nimodipine may be given intravenously for a few days, in the same doses as for aneurysmal SAH (1-2 mg/kg per $\mathrm{h}$ with monitoring of blood pressure) or orally $60 \mathrm{mg}$ every 4-8 h, for 4-12 weeks. The effect this has on the various symptoms and complications is unclear. Thunderclap headaches seem to stop within $48-72 \mathrm{~h}$, but transient ischaemic attacks or even infarction have been reported in patients treated for several days $[1,16]$. A systematic review found no significant difference in outcome between cases treated with calcium-channel blockers versus those that were not [2, 17]. Nimodipine has no effect on ultrasonographic markers of vasoconstriction [2, 11]. Intra-arterial application of nimodipine has been reported in the treatment of RCVS, analogous to intra-arterial nimodipine treatment for refractory cerebral vasospasm after aneurysmal subarachnoid haemorrhage [18]. The conclusion in the report was that intra-arterial application should be considered in RCVS in progressive or treatment-refractory cases, although only as emergency therapy. Further clinical studies are warranted to determine the best possible therapeutic approach [18].

Our report supports the theory of abnormal sympathetic activity, which is observed in $\mathrm{AD}$, as the underlying pathophysiology of RCVS. Schwedt et al. [19] suggested $\mathrm{AD}$ as one factor in the pathophysiology of RCVS. The proximal portions of the large intracranial arteries are innervated by noradrenaline-containing sympathetic afferents, which modulate vascular tone. Abnormal antidromic discharge of sympathetic afferents, i.e. autonomic dysreflexia can cause alterations in vascular tone or acute vasoconstriction and produce head pain. This has hitherto been a theory without actual examples in the clinical setting and, to our knowledge, this is the first report of RCVS associated with AD.

In conclusion, RCVS can occur in the setting of $\mathrm{AD}$ in patients with traumatic cervical cord injury. Prompt recognition of RCVS may be of vital importance to avoid further morbidity in SCI patients.

Conflict of interest None.

\section{References}

1. Ducros A, Boukobza M, Porcher R et al (2007) The clinical and radiological spectrum of reversible cerebral vasoconstriction syndrome. A prospective series of 67 patients. Brain 130:30913101

2. Gerretsen P, Kern RZ (2009) Reversible cerebral vasoconstriction syndrome: a thunderclap headache-associated condition. Curr Neurol Neurosci Rep 9:108-114

3. Calabrese LH, Dodick DW, Schwedt TJ et al (2007) Narrative review: reversible cerebral vasoconstriction syndromes. Ann Intern Med 146:34-44

4. Singhal AB (2004) Postpartum angiopathy with reversible posterior leukoencephalopathy. Arch Neurol 61:411-416

5. Koopman K, Teune LK, Ter Laan M et al (2008) An often unrecognized cause of thunderclap headache: Reversible cerebral vasoconstriction syndrome. J Headache Pain 9:389-391

6. McClain WA, Shields CP, Sixsmith DM (1999) Autonomic dysreflexia presenting as a severe headache. Am J Emerg Med $17: 238-240$

7. Chaves CJ, Lee G (2008) Reversible posterior leukoencephalopathy in a patient with autonomic dysreflexia: a case report. Spinal Cord 46:760-761

8. Yarkony GM, Katz RT, Wu YC (1986) Seizures secondary to autonomic dysreflexia. Arch Phys Med Rehabil 67:834-835

9. Vallès M, Benito J, Portell E et al (2005) Cerebral hemorrhage due to autonomic dysreflexia in a spinal cord injury patient. Spinal Cord 43:738-740

10. Warlow C, van Gijn J, Dennis M et al (2008) Stroke: practical management, 3rd edn. Blackwell, Oxford

11. Chen SP, Fuh JL, Chang FC et al (2008) Transcranial color Doppler study for reversible cerebral vasoconstriction syndromes. Ann Neurol 63:751-757

12. Chen SP, Fuh JL, Lirng JF et al (2006) Is vasospasm requisite for posterior leukoencephalopathy in patients with primary thunderclap headaches? Cephalalgia 26:530-536

13. Call GK, Fleming MC, Sealfon S et al (1988) Reversible cerebral segmental vasoconstriction. Stroke 19:1159-1170

14. Dodick DW (2002) Thunderclap headache. J Neurol Neurosurg Psychiatry 72:6-11

15. Chen SP, Fuh JL, Lirng JF et al (2006) Recurrent primary thunderclap headache and benign CNS angiopathy: spectra of the same disorder? Neurology 67:2164-2169

16. Ducros A, Bousser MG (2009) Reversible cerebral vasoconstriction syndrome. Pract Neurol 9:256-267

17. Gerretsen P, Kern RZ (2007) Reversible cerebral vasoconstriction syndrome or primary angiitis of the central nervous system? Can J Neurol Sci 34:467-477

18. Elstner M, Linn J, Müller-Schunk S, Straube A (2009) Reversible cerebral vasoconstriction syndrome: a complicated clinical course treated with intra-arterial application of nimodipine. Cephalalgia 29:677-682

19. Schwedt TJ, Matharu MS, Dodick DW (2006) Thunderclap headache. Lancet Neurol 5:621-631 